\title{
ROUND THE CORNER
}

Strategies for preventing occupational stress in healthcare workers: past evidence, current problems

Commentary on...Cochrane Corner

https://www.cochranelibrary.com/cdsr/doi/10.1002/14651858.CD002892.pub5/full

Riccardo De Giorgi*

University of Oxford, Department of Psychiatry, Warneford Hospital, Oxford, OX3 7JX, UK

Bianca M. Dinkelaar, Department of Classics, Oxford, OX1 3LU, UK

*riccardo.degiorgi@psych.ox.ac.uk

\section{Biography}

Riccardo De Giorgi is a Wellcome Trust Doctoral Training Fellow at the Department of Psychiatry, University of Oxford, and Honorary Clinical Fellow at Oxford Health NHS Foundation Trust. He works on experimental medicine trials in mood disorders. He is interested in the evidence-based treatment of mental illness and the neuroscientific underpinnings of psychopharmacology.

Bianca M. Dinkelaar holds an MPhil from the Faculty of Classics, University of Oxford, and works on psychological and sociological approaches to ancient philosophy and religion. Her research interests and publication topics include medical ethics and mental health.

\section{Summary}

The problem of occupational stress in healthcare workers is hardly new, but effective interventions in this area are lacking despite being sorely needed - especially in the ongoing Covid-19 pandemic. The review by Ruotsalainen et al, (2015) in the Cochrane Corner includes 58 studies involving 7'188 healthy participants. Its results suggest that cognitive-behavioural therapy and mental and physical relaxation reduce stress more than no intervention but not more than alternative interventions, and that changing work schedules may lead to a reduction of stress. Other organisational interventions showed no effect on stress levels. However, the evidence is of low quality due to risk of bias and lack of precision. This Round the Corner commentary critically appraises the Cochrane review and attempts to put its findings into the current real-world context.

Declaration of interest

None.

What is "occupational stress"?

Stress is a state of mental or emotional strain or tension of which the paraphysiological function is to trigger a fight-or-flight reaction from the body in the face of a threat (NIMH). However, 
long-term stress prevents the body from returning to its normal functioning and is typically characterised by exhaustion, a sense of reduced effectiveness, and decreased motivation, leading to the development of dysfunctional attitudes and behaviours in a range of functional domains (Ruotsalainen, 2015). When stress is either (or both) caused by or expressed in someone's work environment, we consider this "occupational stress". Since occupational stress has both a health and an economic impact, it is in the interest of governments and healthcare institutions to find the most effective ways for avoiding it.

\section{Occupational stress in healthcare workers: an under-researched problem showing a compelling new interest}

Studies have shown that levels of occupational stress in healthcare workers are high, with the UK healthcare sector having one of the highest estimated prevalence rates of work-related stress (HSE 2019). Occupational stress may lead to burnout and psychosomatic illness, and therefore to reduced quality of life in healthcare workers and worse healthcare service provision (Weinberg \& Creed, 2000). This could have a devastating "snowball effect" on the overall health and quality of life of the general public.

Research in this area has hitherto been little and insufficient; however, this issue has recently come to the foreground due to an increased interest in the wellbeing of healthcare workers during the Covid-19 pandemic (Preti, 2020). Nevertheless, amongst the plethora of papers advocating the need for immediate action, the number of concrete intervention strategies proposed remains disappointingly low.

Preventing vs treating stress (and the importance of its causes)

The development of preventive approaches (Box 1) for mental illness is considered the "holy grail" of psychiatric care (Rice-Oxley, 2019): an idyllic goal that is more desirable than treatment itself, yet hard to achieve, especially since we have not been able to determine specific causative factors for most mental disorders. As such, stress (and stress-related disorders) is quite unique in the sense that its source, no matter how complex, can usually be identified: for example, a significant traumatic event in post-traumatic stress disorder. Therefore, they may be avoided through targeted preventive interventions. Indeed, the adoption of preventive strategies in the workplace is advocated as crucial for an effective management of occupational stress (HSE 2003).

The causes of stress in healthcare workers may differ from the causes of stress in other occupations, for example the risk presented by close contact with diseases, the emotional response to contact with death and suffering, and specific organisational problems and conflicts (Ruotsalainen, 2015). We may question from the start whether interventions directed at stressprevention en general can be at all effective when they are not tailored to the specific underlying causes of stress. The Cochrane review authors seem to acknowledge the importance of this issue and therefore aimed to include only studies on healthcare workers; however, it is unclear whether the interventions had been devised with the specific needs of this population in mind.

Moreover, the Covid-19 pandemic has brought with it additional causes of stress for healthcare workers. Not only have workload and personal health risk increased, but healthcare workers are also confronted with societal stigmas: a fear of healthcare workers spreading the virus has led to social ostracism and even harassment (Bouchard 2020); conversely, the promotion of "NHS heroes" has built unrealistic expectations of the duty of healthcare workers to the public (Cox 2020). Thankfully, the increased fame of healthcare professionals has also been 
accompanied by a renewed interest in their mental health: over the past months new research has been published and novel prevention strategies proposed (Blake 2020).

\section{The "PICO" (Box 2) of the Cochrane review}

The objective of the Cochrane Corner review (Ruotsalainen, 2015) was to assess the effectiveness in preventing occupational stress (outcome) in healthcare workers (population) comparing work- and person-directed interventions (intervention) to no intervention or alternative intervention (comparison). Below, we go into the details of this "PICO".

Participants were further defined as healthcare workers who had not received treatment for mental health illness (e.g. burnout, depression, anxiety disorder), which is appropriate for a study that focusses on prevention.

The Cochrane review authors included the following interventions: cognitive-behavioural techniques (person-directed, provide better ways to think, behave, and feel in stressful conditions), relaxation techniques (person-directed, avert attention from stress and build resilience), and organisational interventions (work-directed, reduce the occurrence or impact of stressful events by amending work practices). It is unclear why other potentially useful interventions, such as counselling or psychodynamic therapy (Reynolds, 2000), had not been considered. Mixed interventions, for example cognitive-behavioural plus relaxation techniques, were accounted for. A further distinction between physical and mental relaxation techniques was only described (and analysed accordingly) in the "Results" section, thus appearing as a post-hoc assessment; however, findings were also presented for relaxation techniques as a whole. The authors themselves recognised that for some interventions, such as organising peer-support groups or mentoring schemes, categorisation as either person- or workdirected was hard. We would also argue that pooling or comparing these different interventions can be problematic: physical relaxation techniques such as massage are more easily seen as a treatment rather than a preventive strategy, whereas mental relaxation (e.g. mindfulness), cognitive-behavioural techniques, and organisational interventions are more likely to have both preventive and treatment facets.

The outcomes were assessed at $<1$ month, 1-6 months, and $>6$ months - a sensible choice since the interventions involved would likely show quite different effects at short versus longer follow-ups; yet, the review authors later acknowledged that measuring all outcomes at either less or more than 1 month would be reasonable too.

For the primary outcome, any previously validated self-reported questionnaire evaluating occupational stress or burnout [most commonly, the Maslach Burnout Inventory (Maslach 1996)] was used. Most of the secondary outcomes, whose discussion would go beyond the space of this commentary, similarly involved subjective measures of anxiety and depressive symptoms. The authors deliberately excluded outcomes that, in their opinion, did not directly assess stress or its consequences for individuals; however, we argue that some of these measures, such as absenteeism, could have gauged the interventions' effectiveness very objectively, hence reducing bias issues - particularly with blinding, a very important concern with this kind of research. None of the included studies reported figures of cost-effectiveness, which is unfortunate since these data could more immediately support the implementation of beneficial interventions especially at an organisational level. 
This Cochrane review (Ruotsalainen, 2015) was an update of a previous one (Marine, 2006) by the same research group. Their revised search strategy appears very thorough: more electronic databases were explored, and the additional hand-search comprised reference lists as well as all the issues of the specialist journal "Work\&Stress".

Publication bias was assessed in intervention comparisons containing five or more studies, but the assessment may not have been powered enough as the Cochrane Handbook recommends a minimum of ten studies (Higgins \& Thomas, 2020).

The study types included only randomised controlled trials (RCTs) for cognitive-behavioural and relaxation techniques, whereas for organisational interventions cluster RCTs and prospective cohort studies were considered too. Cross-over trials were not excluded, providing they had a "sufficient wash-out period" according to the Cochrane review authors; however, the very notion of "wash-out" seems questionable for interventions that aim to teach, possibly with lasting efficacy, strategies for preventing stress.

Data was collected, extracted, and analysed by calculating effect sizes (generally, standardised mean difference or SMD) with 95\% confidence intervals (CI), in agreement with best-practice. The review authors made a commendable effort to obtain missing data from numerous studies. Heterogeneity was calculated and considered significative when $>50 \%$. The review authors perplexingly asserted that, in the latter case, the "most likely explanation is that there are data input errors".

The assessment of the risk of bias was performed according to the standard of the time (Higgins \& Green, 2011). Items for "blinding" were not used because none of the included studies did blind participants or intervention-providers and most outcomes were from self-reported questionnaires. The review authors agreed with the studies' researchers in maintaining that "blinding is impossible" in this type of studies; however, we argue that blinding would be "difficult" rather than impossible, particularly with the small sample sizes of the included trials, as demonstrated by other studies employing comparable psychosocial interventions.

Sensitivity analyses (Box 3) were conducted with the exclusion of studies deemed at high risk of bias. Subgroup analysis (Box 3) was performed to differentiate between nurses, physicians, and other healthcare professionals; we believe this subgrouping was scarcely informative, whereas distinguishing between "clinical settings" would lead to more valuable findings, as it is plausible that occupational stress is very dissimilar in, for example, emergency services versus paediatrics or general medicine.

\section{The results of the search}

The literature search yielded a total of 58 records. The studies' flow chart (Figure 1) as well as the main text of the review detail the screening-and-selection process in a somewhat confusing fashion. Some of the studies had been published before the previous Cochrane review from 2006 (Marine, 2006) and yet had not been captured, thus confirming the higher sensitivity of this updated review (Ruotsalainen, 2015). Most of the articles were from after 2010, possibly showing a growing interest in this topic - a trend easily confirmed by performing a similar search today. Three trials (Gomez-Gascon, 2013; Niks, 2013; Spoor, 2010) were ongoing at the time; of these, only the Niks et al (2018)'s results have been made available, reporting the benefit of an organisational intervention on healthcare staff (refer to the paper for further details). 
An adequate number of studies for each outcome's timescale was retrieved (i.e. $<1$ month: 24 studies, 1-6 months: 22 studies, $>6$ months: 12 studies). The review authors may be praised for their industry in distinguishing the (sometimes) ambiguous intervention and comparison arms so that more data could be used for their meta-analyses.

The review's population consisted of 7188 participants, of which 3592 in the various intervention groups and 3596 in the control groups. Sample sizes were very diverse across studies, ranging from $<20$ to $>300$. It is worth noting that, and unclear why, most interventions were directed at nurses, with very few studies involving physicians.

The evidence: do cognitive-behavioural techniques, relaxation techniques, and organisational interventions prevent occupational stress in healthcare workers?

For a detailed report of all pooled results, we refer to the Cochrane review's (Ruotsalainen, 2015) full text. It should be noted that, even for the primary outcome, many different scales were used.

Cognitive-behavioural techniques were more effective in preventing occupational stress compared to no intervention, but not to any alternative intervention, only at follow-ups longer than one month, namely at 1-6 months (8 studies, 549 participants, SMD $-0.38,95 \% \mathrm{CI}-0.59$ to -0.16 ) and even more so $>6$ months ( 2 studies, 157 participants, SMD $-1.04,95 \% \mathrm{CI}-1.37$ to -0.70$)$.

Relaxation techniques showed similar effects already at $<1$ month (4 studies, 97 participants, SMD $-0.48,95 \%$ CI -0.89 to 0.08 ), as well as at $1-6$ months (12 studies, 521 participants, SMD $-0.49,95 \%$ CI -0.78 to -0.21 ) and $>6$ months (1 study, 40 participants, SMD $-1.89,95 \% \mathrm{CI}-$ 2.65 to -1.13 ). Physical relaxation techniques showed a slightly more positive, yet nonsignificative better trend than their mental relaxation counterparts.

Organisational interventions differed very much from each other, therefore the large majority of data could not be pooled for meta-analysis. In their results summary, the Cochrane review authors mentioned two studies showing that a change in work schedules reduced occupational stress; however, we could only retrieve three separate records that reported a significative effect on stress levels for three different interventions - respectively, Ewers et al (2002) (training programme for handling behaviourally-problematic patients, 20 participants, SMD -1.23, $95 \% \mathrm{CI}-2.21$ to -0.26 ;), Bourbonnais et al (2011) (changing working conditions, 488 participants, SMD -0.38, 95\%CI -0.56 to -0.20 ), and Peterson et al (2008), (peer-support groups, 131 participants, SMD $-0.38 ; 95 \% \mathrm{CI}-0.73$ to -0.03 ).

Comparisons between cognitive-behavioural techniques, relaxation techniques, and organisational interventions as compared to any other intervention were mostly hard to calculate, inconsistent, or non-significative different - the latter tentatively suggesting that "doing something...anything" is better than "doing nothing" for preventing occupational stress.

Sensitivity and subgroup analyses, where performed, did not change the outcomes as described.

The quality of the evidence was mainly in the low range, especially due to the elevated risk of bias. Most of the included studies were methodologically poor. However, one single study (Günüsen \& Ustun, 2010) was of high methodological quality thanks to a low risk of bias in randomisation, allocation concealment, incomplete outcome data, and selective reporting - in our view, proving that high-quality studies may be difficult, but still feasible, in this research area. 


\section{Conclusions}

In summary, the interventions included in this Cochrane review (Ruotsalainen, 2015), namely cognitive-behavioural techniques, relaxation techniques, and organisational changes, could be useful for preventing stress in occupational workers - though from a statistical perspective and in the presence of high risk of bias.

The interpretation of the clinical significance of these findings, however, is more challenging. Even the most commonly-used and widely-validated scale for measuring occupational stress, namely the MBI (Maslach 1996), has no generally accepted change regarded as clinically relevant. The Cochrane review authors went through the effort of contacting the MBI's authors, but could not clarify this matter yet - hence, we would reiterate our suggestion for future studies to consider adding proxy outcome measures, such as days of sick leave due to stress. However, the authors correctly used standardised mean differences to gauge the significance of the effect sizes.

The issue of compliance with these interventions, especially if not targeted to the healthcare workers' quite specific needs and circumstances, is in our view of crucial importance.

Overall, the prevention of occupational stress in healthcare workers remains a problem of great relevance, especially in the context of the COVID-19 pandemic; therefore, further research is certainly warranted.

\section{Acknowledgments}

None.

\section{Funding}

None.

\section{Authors contribution}

RDG is responsible for the ideation, design, and write up of the manuscript. BMD is responsible for the literature review and for supporting with the design of the manuscript and its revision.

\section{Boxes, figures \\ Box 1 Prevention}

Prevention is often described in lay terms as a strategy applied to a non-ill population to avoid the onset of that illness in that population; for example, smoking-cessation aids are a preventive measure against lung cancer in people who have not developed lung cancer yet. In epidemiology, however, the concept of prevention is more complex and is subdivided in primary, secondary, and tertiary prevention.

Primary prevention includes any approach that protects the health of people who have not yet become sick - in practice, what we described just above.

Secondary prevention refers to the early recognition and management of a paraphysiological or pathological abnormality that would otherwise almost inevitably lead to a full-blown disorder; for example, reversing high blood pressure to avoid a later myocardial infarction. Arguably, secondary prevention could already qualify as a form of "treatment". 
Tertiary prevention involves measures that curb or sometimes remove the complications of an illness only after that illness has already occurred; for example, physical rehabilitation following a stroke. Again, tertiary prevention can be seen as another form of "treatment".

Box 2 "PICO"

PICO stands for Population, Intervention(s), Comparison(s), Outcome(s). It is the best way to formulate a clear clinical query to which a research study would like to find a meaningful answer. Today, best research practice recommends for a really informative study title and aims to always follow the PICO stricture.

Box 3 Sensitivity versus subgroup analysis

A sensitivity analysis is a statistical tool that allows the interrogation of data with "what if" questions. The resulting findings will not reflect the entirety of the collected data, but may strengthen the validity of the study's results or shed some light on additional research questions. For example, in this Cochrane review the authors asked themselves "what if we only had data from higher quality studies?", therefore they eliminated highly-biased studies from their analysis; their sensitivity analysis results remained in line with the findings from their primary analysis.

A subgroup analysis instead asks the same research question of the primary analysis by probing data for specific participants' subsets of the whole study population. For example, in this Cochrane review the authors performed several additional subgroup analyses to see if the included interventions showed any different efficacy for the groups of nurses, physicians, and other healthcare professionals.

Figure 1 Flow chart

A flow chart or diagram represents the best practice, according to the Preferred Reporting Items for Systematic Reviews and Meta-Analyses (PRISMA) guidelines, for describing the process that led from the initial literature search to the choice of studies included in the final analysis. Today, all well-performed systematic reviews and meta-analysis would report a PRISMA flow chart (usually named "figure 1") in the body of the article. A detailed flow chart should allow any researcher to replicate the findings, in terms of the included articles, of the systematic review at a specified time.

A PRISMA flow chart is usually composed of at least four rows (refer to the example in the figure). The first row ("identification") often depicts two boxes: one containing the number of records retrieved via electronic databases and for those added through a manual search. These are often merged in a single box, with duplicate records from different databases removed. The second row ("screening") should have a box with the number of records whose title and abstract have been screened, according to pre-specified inclusion/exclusion criteria, and a box with those records excluded on this basis, with reason. The third row ("eligibility") replicates what was done in the second row, but this time the full-text of the articles is assessed. Finally, the fourth row ("Included") reports the number of studies that will eventually be analysed. 


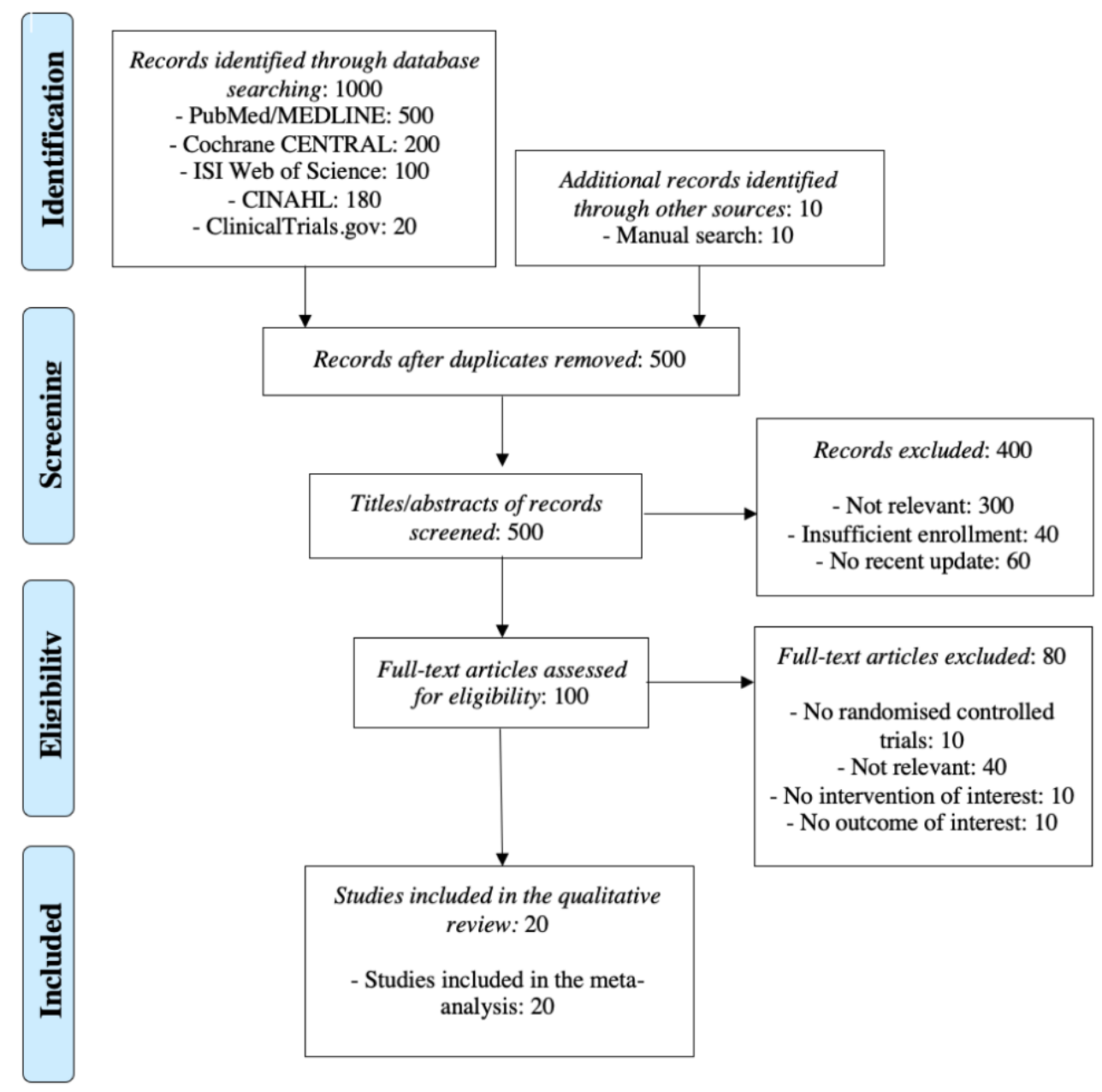

\section{References}

Blake H, Bermingham F, Johnson G, Tabner A (2020) Mitigating the Psychological Impact of COVID-19 on Healthcare Workers: A Digital Learning Package. Int J Environ Res Public Health; 17(9):2997. doi: 10.3390/ijerph17092997.

Bouchard JP (2020) Covid-19: les soignants entre héroïsation et ostracisation [Covid-19: health care workers between heroism and ostracism]. Rev Infirm; 69(260-261):31-32. French. doi: 10.1016/S1293-8505(20)30149-4.

Bourbonnais R, Brisson C, Vinet A, Vézina M, Abdous B, Gaudet M (2006) Effectiveness of a participative intervention on psychosocial work factors to prevent mental health problems in a hospital setting. Occupational and Environmental Medicine; 63(5):335-42.

Cox CL (2020) 'Healthcare Heroes': problems with media focus on heroism from healthcare workers during the COVID-19 pandemic. $J$ Med Ethics; 46(8):510-513. doi: 10.1136/medethics-2020-106398.

Ewers P, Bradshaw T, McGovern J, Ewers B (2002) Does training in psychosocial interventions reduce burnout rates in forensic nurses? Journal of Advanced Nursing; 37(5):4706. 
Gomez-Gascon T, Martin-Fernandez J, Galvez-Herrer M, Tapias- Merino E, Beamud-Lagos M, Mingote-Adan JC (2013) Effectiveness of an intervention for prevention and treatment of burnout in primary health care professionals. BMC Family Practice;14(1):173.

Günüsen N, Ustun B (2010) An RCT of coping and support groups to reduce burnout among nurses. International Nursing Review; 57(4):485-92.

Health and Safety Executive (2003) Beacons of excellence in stress prevention. Internet, accessed $5^{\text {th }}$ October 2020, https://www.hse.gov.uk/research/rrpdf/rr133.pdf.

Health and Safety Executive (2019) Work-related stress, anxiety or depression

statistics in Great Britain. Internet, accessed $5^{\text {th }}$ October 2020 https://www.hse.gov.uk/statistics/causdis/stress.pdf.

Higgins JPT, Green S (editors) (2011). Cochrane Handbook for Systematic Reviews of Interventions, Version 5.1.0. The Cochrane Collaboration.

Higgins JPT, Thomas J (editors) (2020). Cochrane Handbook for Systematic Reviews of Interventions, Version 6.1. The Cochrane Collaboration. Available from www.cochranehandbook.org.

Marine A, Ruotsalainen Jani H, Serra C, Verbeek JH (2006). Preventing occupational stress in healthcare workers. Cochrane Database of Systematic Reviews. doi: 10.1002/14651858.CD002892.pub2

Maslach C, Jackson SE, Leiter SP (1996). Maslach Burnout Inventory Manual, 3rd edition. Palo Alto, CA: Consulting Psychologists Press.

Niks IMW, De Jonge J, Gevers JMP, Houtman ILD (2013). Design of the DISCovery project: tailored work-oriented interventions to improve employee health, well-being, and performance-related outcomes in hospital care. BMC Health Services Research; Vol. 13.

National Institute of Mental Health (2020). 5 Things You Should Know About Stress. NIH Publication 19-MH-8109. Internet, accessed $5^{\text {th }}$ October 2020, https://www.nimh.nih.gov/health/publications/stress/index.shtml.

Niks IMW, de Jonge J, Gevers JMP, Houtman ILD (2013) Design of the DISCovery project: tailored work-oriented interventions to improve employee health, well-being, and performance-related outcomes in hospital care. BMC Health Services Research; Vol. 13.

Niks IMW, de Jonge J, Gevers JMP, Houtman ILD (2018) Work Stress Interventions in Hospital Care: Effectiveness of the DISCovery Method. Int J Environ Res Public Health; 13;15(2):332. doi: 10.3390/ijerph15020332.

Peterson U, Bergstrom G, Samuelsson M, Asberg M, Nygren A (2008) Reflecting peer-support groups in the prevention of stress and burnout: randomized controlled trial. Journal of Advanced Nursing;63(5):506-16.

Preti E, Di Mattei V, Perego G, Ferrari F, Mazzetti M, Taranto P, Di Pierro R, Madeddu F, Calati R (2020). The Psychological Impact of Epidemic and Pandemic Outbreaks on 
Healthcare Workers: Rapid Review of the Evidence. Curr Psychiatry Rep; 22(8):43. doi: 10.1007/s11920-020-01166-z.

Reynolds S (2000). Interventions: what works, what doesn't? Occup Med; 50(5):315-9. doi: 10.1093/occmed/50.5.315.

Rice-Oxley M (2019). Prevention: the new holy grail of treating mental illness. The Guardian, Internet, accessed 5th October 2020, https://www.theguardian.com/society/2019/jun/08/prevention-the-new-holy-grail-of-treating$\underline{\text { mental-illness }}$

Ruotsalainen JH, Verbeek JH, Mariné A, Serra C (2015) Preventing occupational stress in healthcare workers. Cochrane Database Syst Rev; doi: 10.1002/14651858.CD002892.pub5..

Spoor E, De Jonge J, Hamers JP (2010) Design of the DIRECT- project: interventions to increase job resources and recovery opportunities to improve job-related health, well-being, and performance outcomes in nursing homes. BMC Public Health; 10:293.

Weinberg A, Creed F (2000) Stress and psychiatric disorder in healthcare professionals and hospital staff. Lancet; 355(9203):533-7. 